Chapter 4

\title{
Cranial Nerves and Nerve Surgery in the Oral and Maxillofacial Region
}

\author{
Shahram Nazerani and Tina Nazerani \\ Additional information is available at the end of the chapter \\ http://dx.doi.org/10.5772/63080
}

\begin{abstract}
The head and neck surgeon is confronted with cranial nerves in the course of operations and he or she must know the anatomy and the ways to treat complications should they happen. In this chapter we focus on the subject of cranial nerves and begin with the history and anatomy and then to individual nerves and maladies of these nerves and complications of surgical procedures involving these nerves.
\end{abstract}

Keywords: cranial nerves, cranial nerve disorders, cranial nerve surgery, nerve repair, nerve transfer

\section{Introduction}

Ancient Egyptian mummification artisans had no consideration for the brain; the internal organs such as the liver, intestines, lung, and heart were preserved in separate jars; they broke the skull bone through the nose with a tool like a hook and a tool to blend lift the brain and then allowed it to drain out the nose or flushed it out with water. The Egyptians thought the heart was the site of bravery and gave no importance to the brain for afterlife preservation (Figure 1).

The history regarding exploration of the cranial nerves and their anatomy is ancient. Galen's classification of the cranial nerves, excluding the olfactory nerve, was composed of seven pairs; the sixth pair included the glossopharyngeal, vagus, and accessory nerves all traveling through the jugular foramen [1].

The cranial nerves were essentially identified and numbered based on the opening through which they exited the skull base. Our knowledge of the cranial nerves grew more clearly by 
medieval Middle Eastern and European scholars such as Rhazes, Avicenna, Jorjani, Mundinus, Benedetti, Achillini, Massa, and Berengario deCarpi [2].

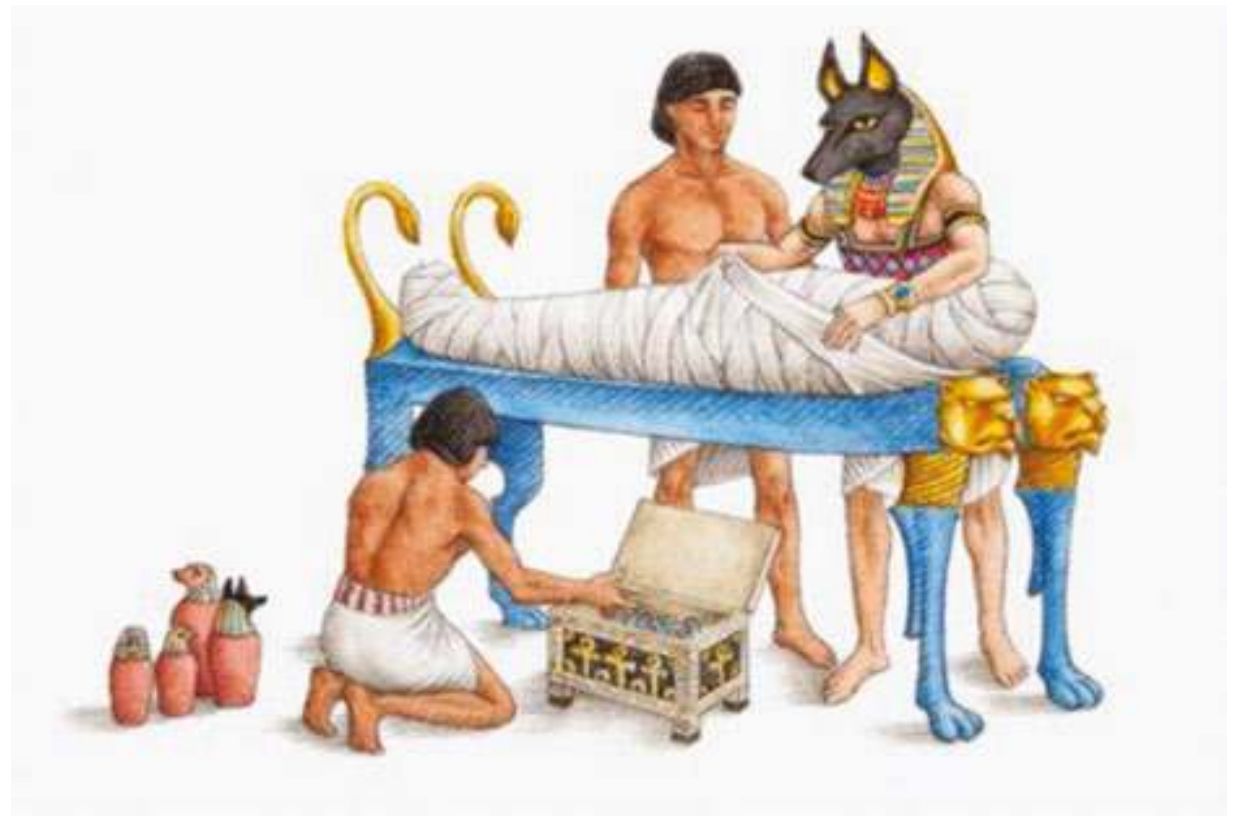

Figure 1. Mummification process with internal organs set aside in jars.

Italian anatomists in the fourteenth and fifteenth centuries identified the olfactory nerve as a cranial nerve. The work of these anatomists laid the foundation for the doctoral thesis of the German anatomist Samuel Sömmerring (1755-1830 AD), who in 1778 classified the 12 cranial nerves as we recognize them today $[3,4]$. In his thesis, Sömmerring made no meaningful anatomical discoveries, and his classification is essentially no less different from previous anatomists. Nonetheless, the Sömmerring system was rapidly adopted across continental Europe, although it was only slowly accepted in England [5].

\section{Anatomy and naming of the cranial nerves}

The cranial nerve nomenclature is an ordinal system introduced presumably by Galen, by which the nerves are named by their location on the undersurface of the brain, this system has stood the test of time and has not been changed during the ages, although the olfactory nerve has been mentioned even before Galen's ordinal classification. Modern anatomists argue that the olfactory and optic nerves are tracts and not true cranial nerves and should be removed from the "twelve-nerve" classification system [5]. To memorize these nerves, several mnemonics have been devised (Figure 2). 


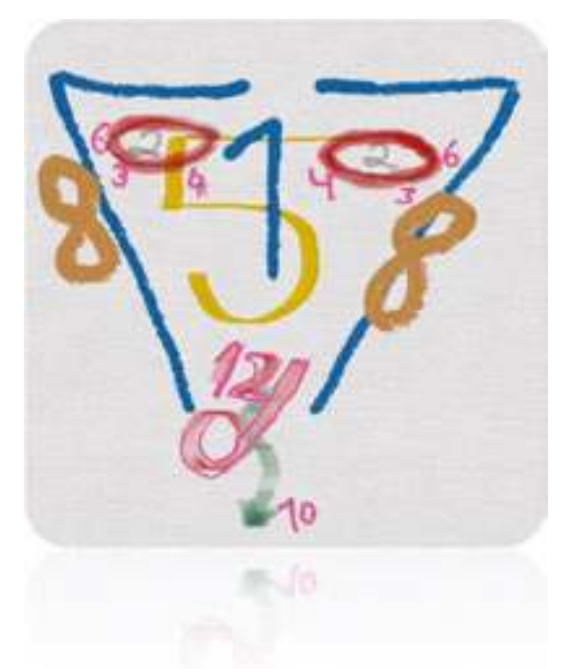

Figure 2. A mnemonic of the cranial nerves.

Galen's classification of cranial nerves is depicted below; he identified seven pairs but he assigned no names to them. The modern classification is shown below, Galen identified seven pairs of nerves, and the interesting point is that the old scholars saw the brain as a whole and not two hemispheres of an organ.

Modern classification:

I. Olfactory nerve

II. Optic nerve

III. Oculomotor nerve

IV. Trochlear nerve

V. Trigeminal nerve

VI. Abducens nerve

VII. Facial nerve

VIII. Vestibulocochlear nerve

IX. Glossopharyngeal nerve

$X$. Vagus nerve

XI. Accessory nerve

XII. Hypoglossal nerve 


\section{Surgical importance of cranial nerves}

There are 12 cranial nerves, but only some of the nerve problems were identified and reported in the early ages. Of the 12 cranial nerves, the trigeminal and facial nerves have been under more scrutiny: the trigeminal due to its excruciating pain and the facial nerve due to its characteristic facial disfigurement in nerve palsy (Figure 3).

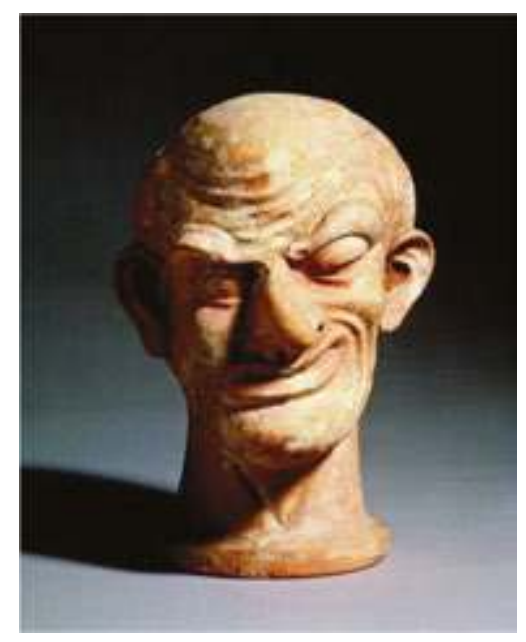

Figure 3. A sculpture of facial palsy.

Other cranial nerves are usually noticed when traumatized, involved in tumoral conditions or injured during an operation.

Multiple cranial nerves are usually involved in procedures such as carotid endarterectomy, anesthesia, and cancer surgery. In a review article, Thiruvenkatarajan et al. investigated cranial nerve injury (CNI) during anesthesia and they found that cranial nerve injuries are unusual complications of supraglottic airway use. Branches of the trigeminal (V), glossopharyngeal (IX), vagus (X), and hypoglossal (XII) nerves may be injured. Lingual nerve (LN) injury was the most commonly reported, followed by recurrent laryngeal, hypoglossal, glossopharyngeal, inferior alveolar, and infraorbital. The culprit is usually poor technique and inappropriate pressure on the nerves due to wrong size or misplacement or overinflation of the cuff. Injury to the recurrent laryngeal nerve (RLN) is usually more long lasting than other nerves involved [6]. In a multicenter prospective study, Fokkema et al. investigated the prevalence of CNI in carotid endarterectomy; 6878 patients were included for analyses. CNI rate at discharge was $5.6 \%$. Sixty patients $(0.7 \%)$ had more than one nerve affected. The hypoglossal nerve was most frequently involved $(2.7 \%)$, followed by the facial $(1.9 \%)$, the vagus $(0.7 \%)$, and the glossopharyngeal $(0.5 \%)$ nerve. The vast majority of these CNIs were transient; only 47 patients $(0.7 \%)$ had a persistent CNI at their follow-up visit [7]. Isolated cranial nerve injuries are herein with special attention to the nerves more prone to injury during maxillofacial surgery. 


\subsection{The olfactory nerve}

The first cranial nerve is composed of receptor neurons which connect the nasal cavity to the brain (Figure 4). The nerve dendrites reside in the nasal cavity on the apical side and the axons pass through the cribriform plate of the skull into the olfactory area of the brain [8].

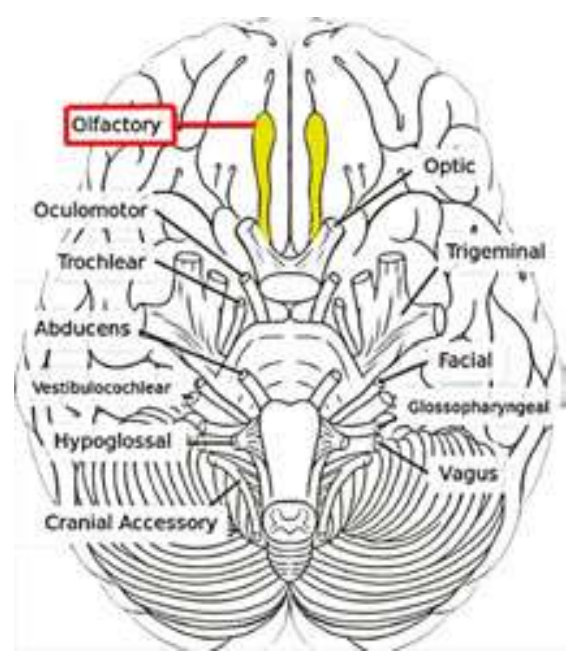

Figure 4. Skull base schematic diagram and the olfactory nerve marked in yellow.

Esthesioneuroblastoma (ENB) is a rare malignant neoplasm arising from the olfactory neuroepithelium. ENB constitutes only 3\% of all malignant intranasal neoplasms. Because of the rarity, the number of patients of ENB treated in individual departments is small. Most of these patients present in locally advanced stages and require multimodality surgery, chemotherapy, and radiotherapy [9]. Impairment of smell may occur following injury to any portion of the olfactory tract, from the nasal cavity to brain. A thorough understanding of the anatomy and pathophysiology combined with comprehensively obtained history, physical exam, olfactory testing, and neuroimaging may help to identify the mechanism of dysfunction and suggest possible treatments. Although most olfactory deficits are neuronal mediated and therefore currently unable to be corrected, promising technology may provide novel treatment options for those most affected. Until that day, patient counseling with compensatory strategies and reassurance is essential in this unique and challenging patient population [10].

\subsection{Optic nerve}

Optic nerve injury may have several etiologies such as tumor, trauma, and inflammation, but in the context of maxillofacial surgery, trauma to the optic nerve is the most important (Figure 5); two types of trauma to the optic nerve are seen, primary and secondary; injury to the nerve fibers and/or vascular supply can be due to direct injury at the time of trauma or secondary as the result of compromised blood supply to the nerve. 


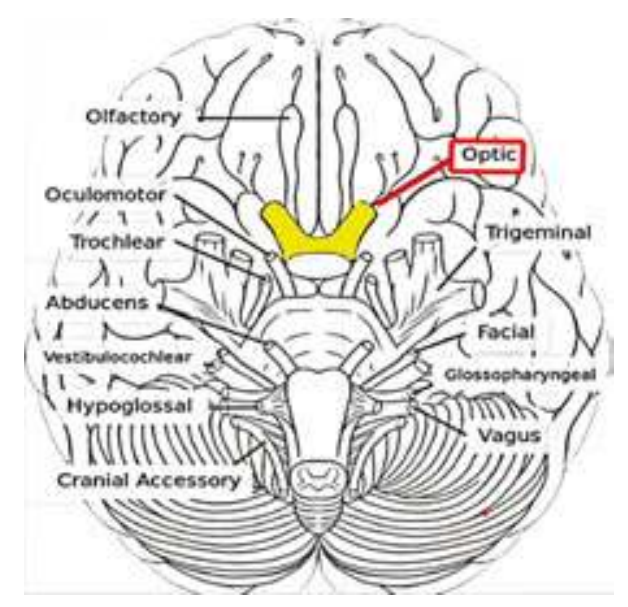

Figure 5. Optic nerve marked in yellow.

\subsection{Oculomotor nerve}

Injuries to the oculomotor nerve present with nerve palsy manifestations (Figure 6); the etiologies can be sellar chordoma, odontogenic abscess, non-aneurysmal subarachnoid hemorrhage, polycythemia, sphenoiditis, brucellosis, interpeduncular fossa lipoma, metastatic cancer, and blood and lymph cancers. Surgical options are correction of nerve palsy, i.e., strabismus surgery. New globe fixation procedures may include fixation to the medial orbital wall, apically based orbital bone periosteal flap fixation, and the suture/T-plate anchoring platform technique [11].

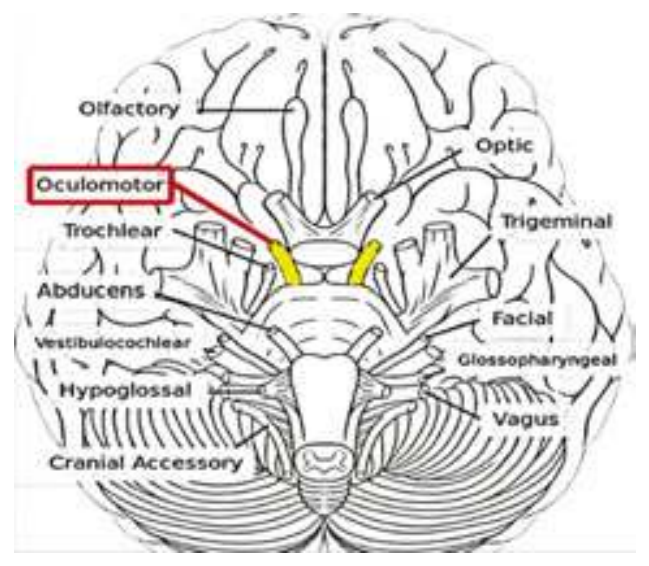

Figure 6. The third cranial nerve marked in yellow. 


\subsection{Trochlear nerve}

The trochlear nerve originates from the trochlear nuclei in the caudal midbrain (Figure 7) and carries primarily motor fibers destined for the superior oblique muscle; the nerve may be encountered in many areas such as the supracerebellar, middle cranial fossa, parasellar, and orbital regions. This nerve was the last cranial nerve found, due its small size and lack of fixation techniques in the earlier periods of anatomical studies. Trauma, tumors, viral infection, and surgery can all injure this nerve with impaired eyeball movement.

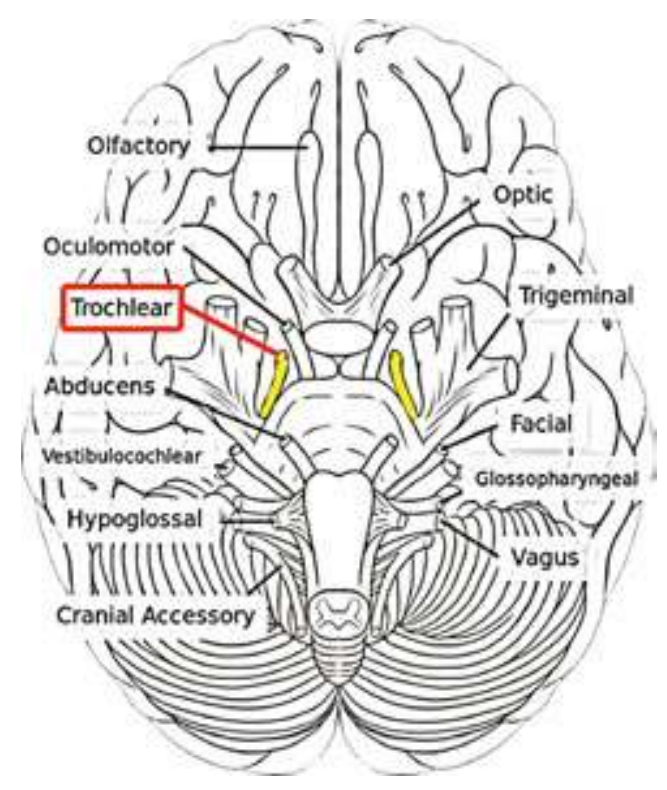

Figure 7. The fourth cranial nerve seen from undersurface of the brain.

\subsection{Trigeminal nerve}

The trigeminal nerve is the largest and most complex of the 12 cranial nerves (Figure 8). The three branches of the trigeminal nerve (the ophthalmic, maxillary, and mandibular branches exit the skull through three separate foramina, namely, the superior orbital fissure, the foramen rotundum, and the foramen ovale, respectively). The mandibular branch had mixed sensory and motor neurons and of all the branches lingual and inferior alveolar are more prone to injury during maxillofacial operations. Injury to the LN and/or inferior alveolar nerve (IAN) is a known complication associated with several oral and maxillofacial surgical procedures. Bagheri et al. in a retrospective study have shown that microsurgical repair of LN and IAN injury has the best chance of successful restoration of acceptable neurosensory function if done within 9 months of the injury. As in all other nerves, the likelihood of recovery decreases progressively when the repair is done more than 9 months after injury [12]. 


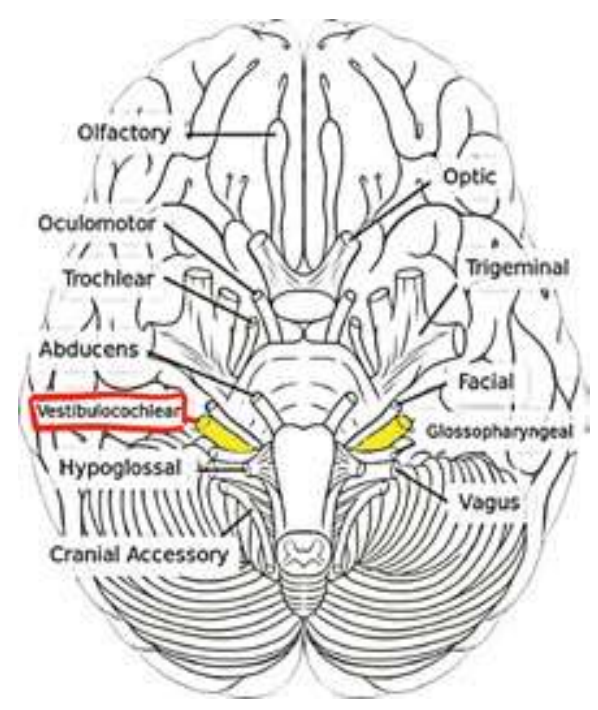

Figure 8. The fifth cranial nerve, the largest cranial nerve.

\subsection{Abducens nerve}

The sixth cranial nerve is a motor nerve with diplopia as its presenting symptom when injured (Figure 9). Binocular diplopia occurs from misalignment of the eyes. The fixation object is imaged onto the fovea of one eye and a non-foveal region of the misaligned eye, creating diplopia. The nerve can be injured in head trauma, autoimmune diseases, and several other conditions. Treatment options include ocular occlusion, mono-vision optical correction, prism glasses, strabismus surgery, and chemo-denervation.

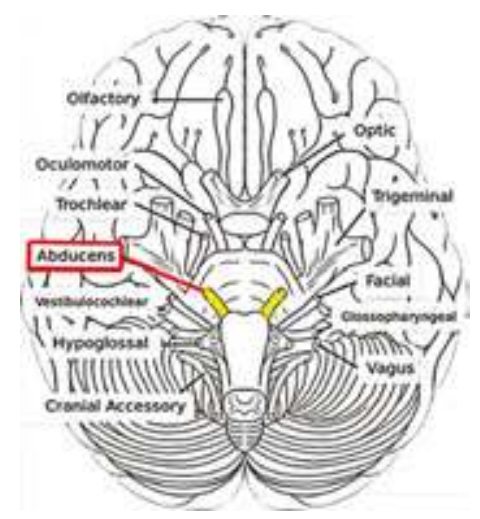

Figure 9. The sixth cranial nerve. 


\subsection{Facial nerve}

Facial nerve paralysis involves orifice control for the eye, nose, and mouth, as well as facial expression (Figure 10). The lack of orifice control for the eye can lead to corneal exposure, keratopathy, and potential visual loss. The orifice control relating to the nose can cause difficulties in breathing with lack of normal opening of the involved nostril.

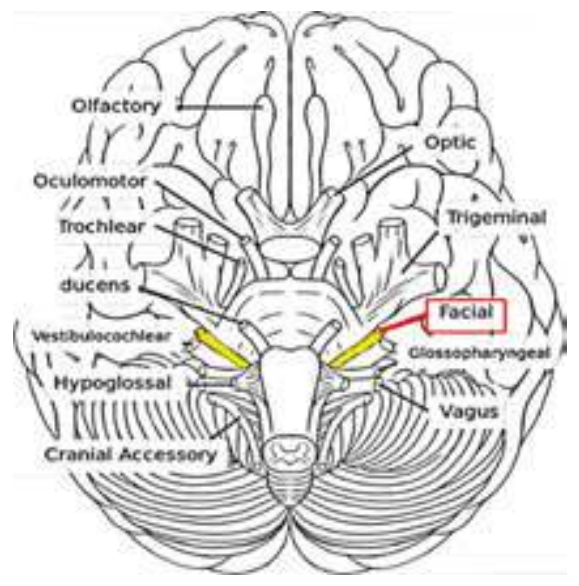

Figure 10. The seventh cranial nerve.

The lack of orifice control for the mouth can affect the symmetry of the face with drooping of the involved side, as well as problems related to speech, chewing, and oral competence leading to drooling. In some cases, the lack of dental protection can lead to dental decay. Some partial facial palsies are seen as attractive (Figure 11).

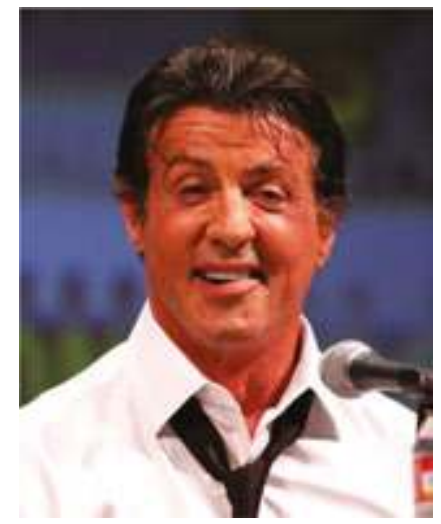

Figure 11. Sylvester Stallone with his "trademark smile." 
The mimetic function, however, of the facial nerve is critical for social interactions. Nonverbal communication is conveyed by facial expression and is essential for normal interpersonal interactions. A smile invokes a smile in others and conveys feelings that cannot be transmitted in any other way. Consequently, a spontaneous dynamic smile is critical for personal interactions. Treatment modalities in facial paralysis and associated movement disorders are numerous and vary based on individual needs and preferences.

\subsubsection{Congenital facial paralysis}

The anatomical presentation of developmental facial paralysis can be summarized into four categories:

1. Aplasia or hypoplasia of cranial nerve nuclei

2. Nuclear agenesis

3. Peripheral nerve abnormalities

4. Primary myopathy [13]

\subsubsection{Acquired facial paralysis}

Three stages of facial nerve paralysis are acute, intermediate, and chronic; the treatment modalities are discussed below.

\subsubsection{Acute facial paralysis}

Identifiable causes of acute paralysis are treated with appropriate medical therapy, following proper identification of the cause. In rare instances, surgical intervention may be necessary to control infection and/or swelling around the facial nerve. In trauma or resection of cancer invading the facial nerve, several reconstructive options are available. These minimize the sequelae of paralysis, optimize immediate patient recovery, and promote the return of facial nerve function.

\subsubsection{Intermediate facial paralysis}

During this stage ( 3 weeks to 2 years), facial nerve recovery is monitored with serial electrophysiological studies, which provide useful prognostic data. In a setting of poorly recovering facial nerve, several procedures can be considered to restore facial appearance and rehabilitate function around the eye and mouth. In the early stages gold weight placement to aid upper eyelid closure (lagophthalmos) and static sling suspension of the midface and lip can be performed with minimal associated downtime. Lagophthalmus (Figures 12 and 13) can be treated by a range of techniques, including tarsorrhaphy, facial slings, and canthopexies. Gold plates provide a solution for temporary or permanent lagophthalmos resulting from facial paralysis. Amer et al. studied the use of gold plates in two different positions in the upper lids. 


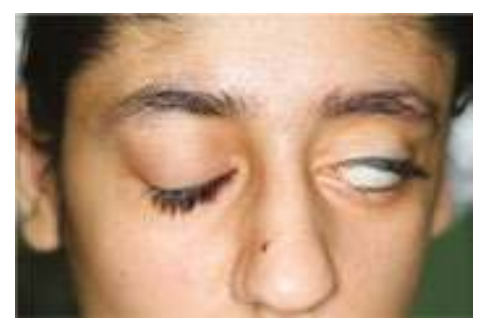

Figure 12. Bell's palsy and lagophthalmos.

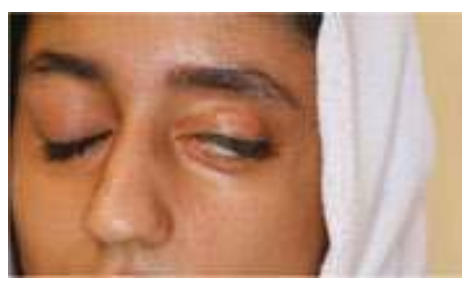

Figure 13. Gold plate inserted, but is not esthetically appealing.

They concluded that gold plate insertion at a higher than usual place of insertion can reduce the drawbacks of lower placement such as "plate show," thinning of the skin over the plate [14].

These procedures do not interfere with the recovering facial nerve. In the later stages, if the facial nerve continues to display poor recovery on EMG, consideration is given to nerve transfer procedures designed to maintain neurological input of facial muscles. A graft from a nearby nerve, most commonly the hypoglossal, can provide such input. Terzis and Karypidis show that cross-facial nerve grafting and concomitant mini-hypoglossal transfer are the procedures that yield higher improvement in blink scores and ratios compared with the rest of the dynamic procedures. Direct orbicularis oculi muscle neurotization achieves a fair blink improvement [15].

\subsubsection{Chronic facial paralysis}

\subsubsection{Paralysis}

Management of chronic facial paralysis, more than two years, depends on numerous factors, including patient preferences, age, and desires. Medical considerations may also limit the available procedures. Reconstructive options range from static suspensions to reanimate via muscle transfer to the paralyzed side.

Static slings such as native fascia (5) or permanent sutures (6) are the easiest methods in facial reanimation. These slings hold the paralyzed side in midline and help hold the lips and prevent 
drooling to a degree. Static slings relax and descend over time, thus potentially requiring additional tightening [16, 17] (Figures 14-18).

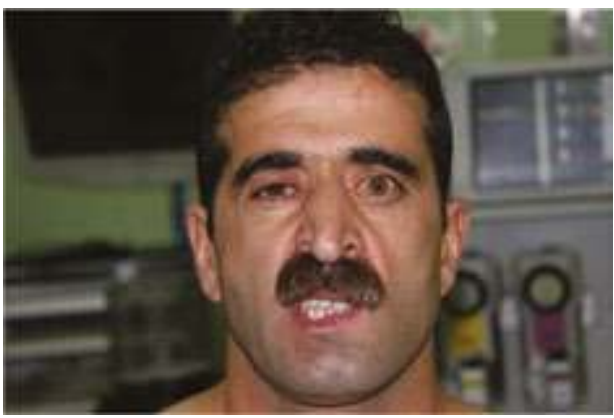

Figure 14. Chronic Bell's palsy, first-stage sural nerve transfer already done.

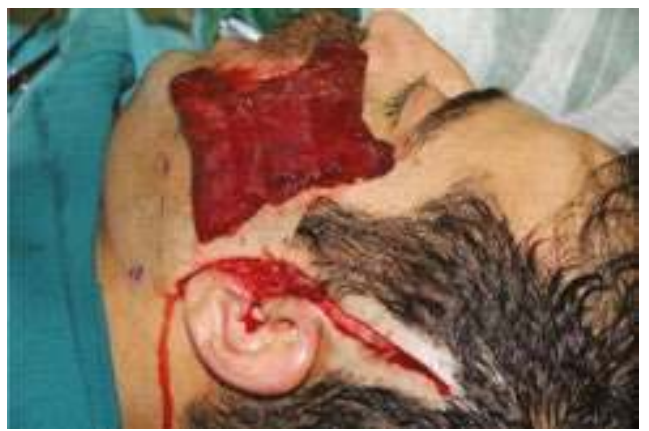

Figure 15. Gracilis muscle bisected and ready for insertion.

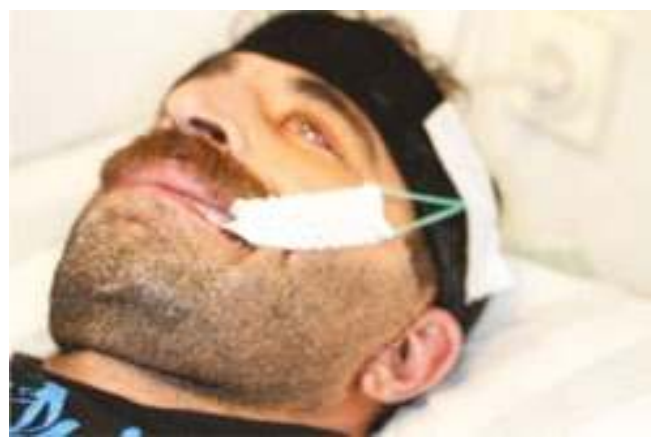

Figure 16. Two months after the operation and the static splint for maintaining the muscle suture. 


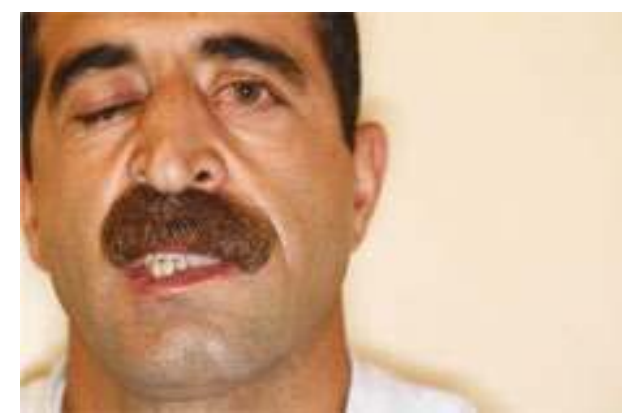

Figure 17. Four months after operation, minimal muscle function has returned; lagophthalmos will be addressed later.

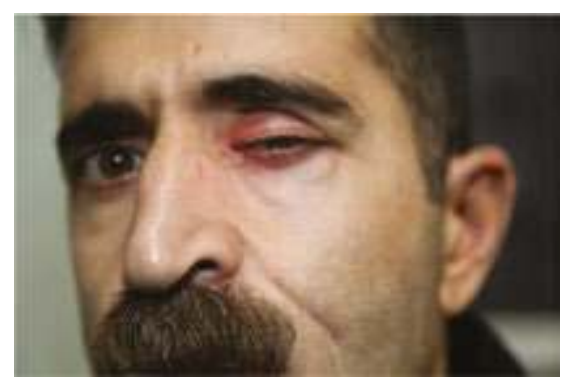

Figure 18. Tendon transfer for the eye with the face contour nearly normal.

One- or two-stage free vascularized muscle transfers are the best options for facial reanimation but they are lengthy operations and more sophisticated than the static slings. Interest in the temporalis muscle transfer has been renewed but the results are inferior to the muscle transfers [18] (Figures 19 and 20).

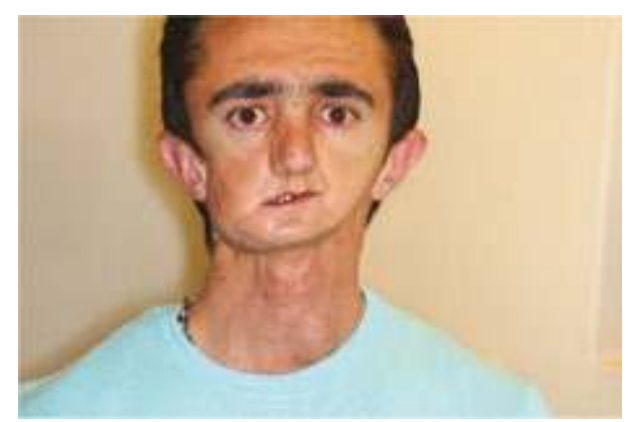

Figure 19. Facial palsy in a patient after reconstruction of the mandible due to childhood radiation-induced lower face atrophy. 


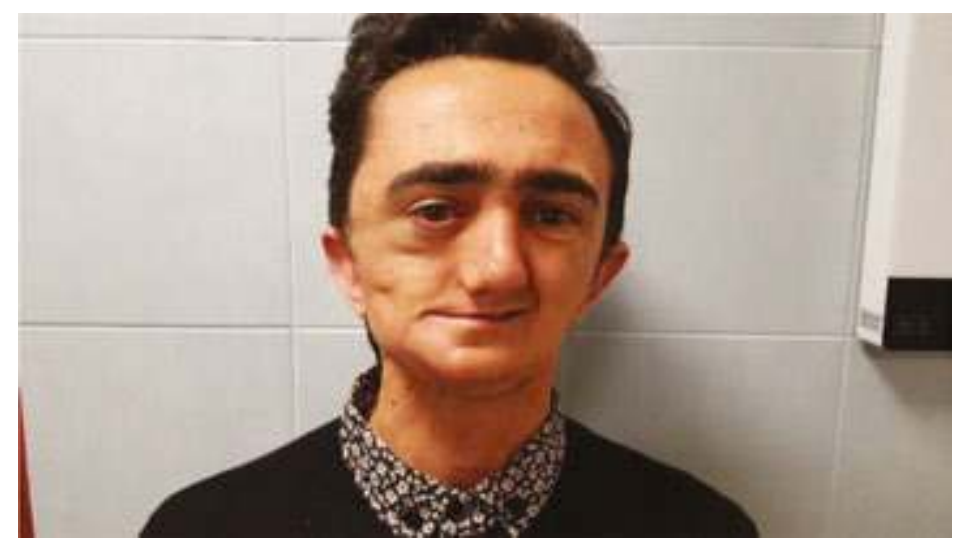

Figure 20. Temporalis transfer for right facial palsy.

The procedure of choice to regain involuntary smile is a two-stage transfer of the gracilis muscle. There are several candidate muscles in the literature, such as the pectoralis minor and latissimus dorsi (LD) as a one-stage muscle transfer (Figure 21).

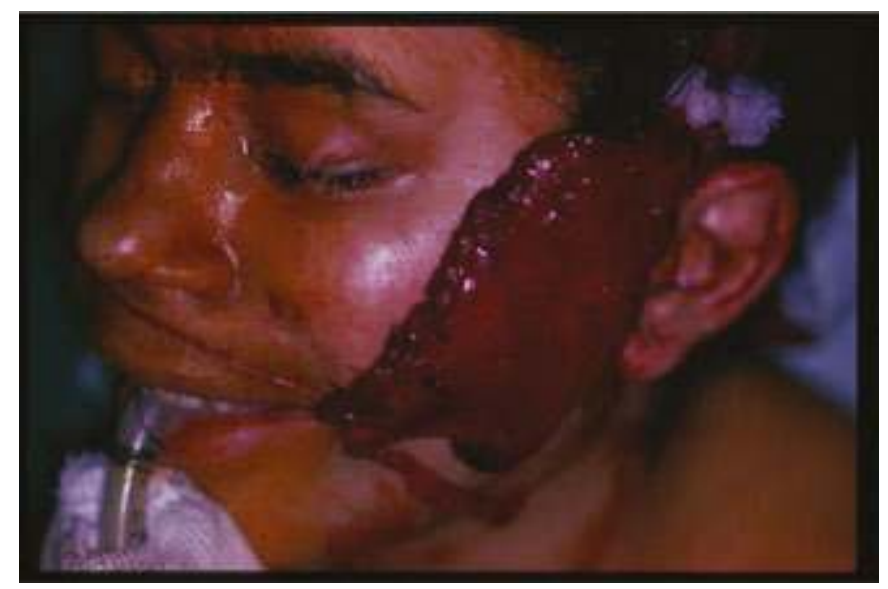

Figure 21. The one-stage LD muscle transfer, which can be done in one stage due to its long nerve.

At the first operation, a branch of the facial nerve on the healthy side is grafted and carried across by a sural nerve graft to the paralyzed side. The recommended route for nerve graft is across the face, through the upper lip and actually lying in the midface area, where at the second stage, a skin elevation is needed to insert the flap. Due to hazardous route of the cable graft, we think that a submental or frontal route is better suited for this operation because the nerve graft is safe when elevating the flaps at the second stage and also in future revision surgeries (Figure 22). 


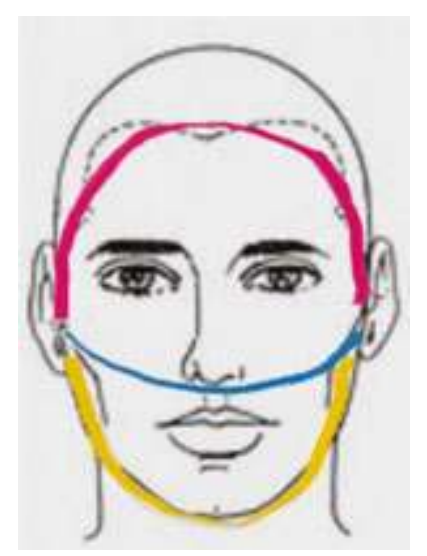

Figure 22. Blue is the recommended route; yellow and red routes are our preferred ways to transfer the nerve graft from one side of the face to the other side.

Six to nine months later, a segment of the gracilis muscle is transferred to the face and connected to the grafted nerve. The muscle becomes functional, providing movement on the paralyzed side. The gracilis transfer affords a better precision to the smile angle and greater movement of the commissure, when compared to temporalis transfer.

Terzis and Anesti used platysma muscle transfer to augment the function of or regulate the overactive previously transferred free vascularized muscle for oral sphincter control [19].

\subsubsection{Synkinesis}

Synkinesis is abnormal involuntary facial muscle movement during the voluntary movement of different muscle groups. For example, eye closure can result in simultaneous contraction of orbicularis oris or platysma contraction during smile. Other cranial nerves such as ocular and abducens have also synkinesis problems.

The problem seems to be the random growth or "miswiring" of facial nerve.

Management is a multimodality protocol which includes multiple session chemo-denervation with botulinium toxin and biofeedback facial muscle retraining and also surgical procedures such as small nerve graft or repair [20].

Choi et al. in a study of botulinum toxin injection in facial paralysis showed significant suppression of synkinesis and improvement of facial symmetry with resulting elevated quality of life, social interaction, personal appearance, and food intake [21].

Radiofrequency ablations are theoretically capable of reducing the injection sessions but are still in investigative stages. At present the best method of treatment for synkinesis is chemodenervation with physical therapy [22-24]. 


\subsection{Vestibulocochlear nerve}

The vestibulocochlear nerve (eighth cranial nerve) is a pure sensory intracranial nerve (Figure 23). This nerve has two functions and hence two nerves: sound transmission and balance. The nerves originate from sensory receptors of internal ear to the brain stem and thence to the post-central gyrus and auditory cortex. The two most frequent etiologies of eighth nerve pathology are skull base trauma and vestibular schwannomas with the latter being the most common lesion.

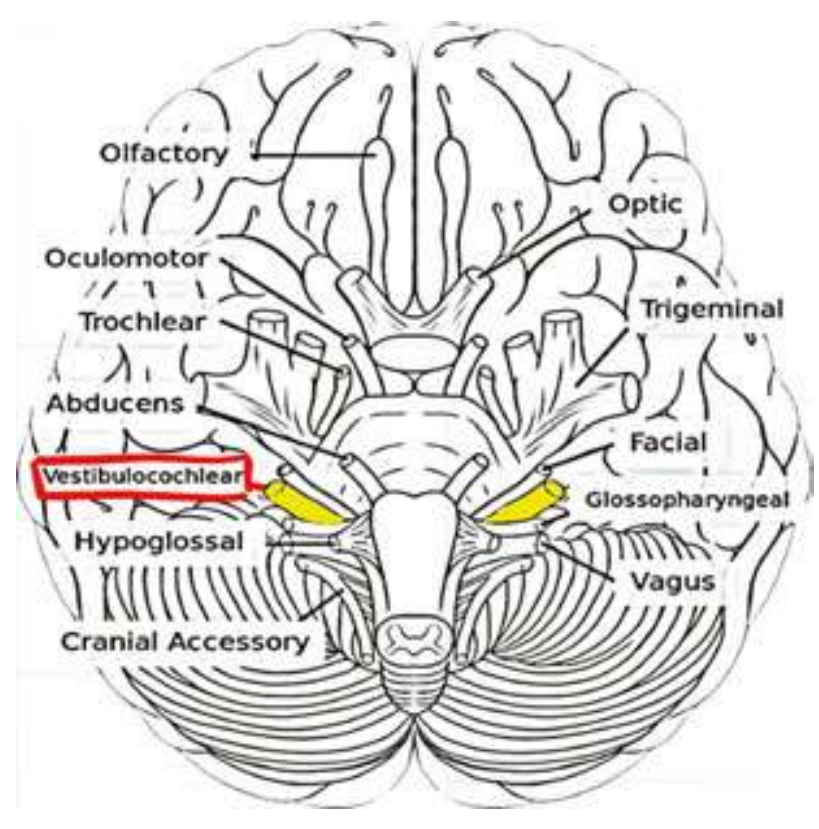

Figure 23. The eighth cranial nerve.

\subsection{Glossopharyngeal}

The ninth cranial nerve or glossopharyngeal nerve is a mixed nerve consisting of both sensory and motor nerve fibers (Figure 24). The origins of sensory fibers are pharynx, middle ear, posterior one-third of the tongue (including taste buds), and the carotid body and sinus. The motor fibers terminate at the parotid gland, the glands of the posterior tongue, and the stylopharyngeus muscle. Hwang et al. in a review article found that frequency of communication between the facial nerve and the vestibulocochlear nerve was the highest $(82.3 \%)$ and the frequency of communication between the facial nerve and the glossopharyngeal nerve was the lowest (20\%). Surgeons should be aware of the nerve communications, which are important during clinical examinations and surgical procedures of the facial nerves such as those communications involved in facial reconstructive surgery, neck dissection, and various nerve transfer procedures [25]. 


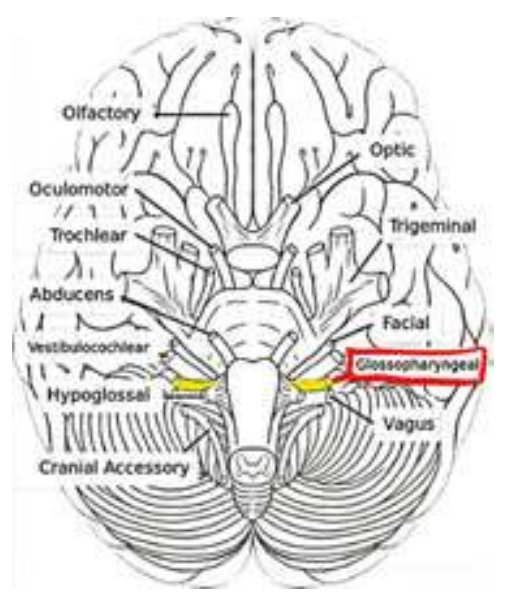

Figure 24. Ninth cranial nerve location at the undersurface of the brain.

The glossopharyngeal nerve is in danger of iatrogenic injury during tonsillectomy and the bilateral injury can be devastating [26].

\subsection{Vagus nerve}

The vagus nerve (Figure 25), from the Latin root meaning "wanderer," is the longest cranial nerve with far reaching functions, from vocal cords to the heart and finally the stomach and gallbladder. This nerve is at greatest risk in head and neck re-exploration.

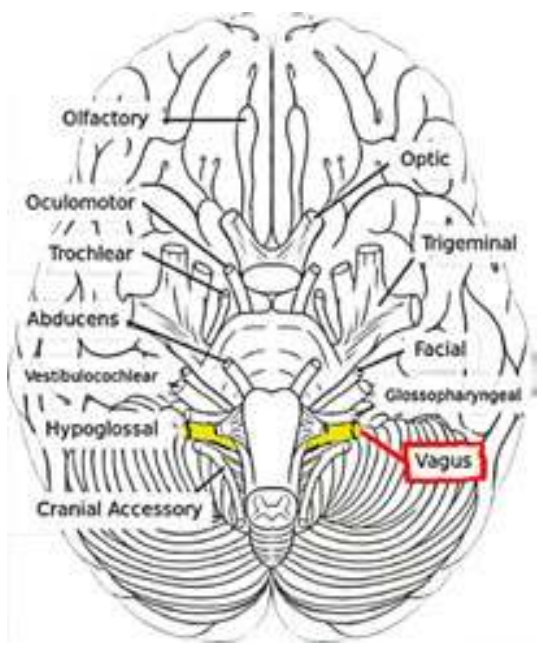

Figure 25. The 10th and the longest cranial nerve. 
One of the important branches of the vagus nerve is RLN, the nightmare of head and neck surgery and especially thyroid surgery. Unrecognized RLN injury entails delayed phonosurgical intervention and laryngeal reinnervation.

Unilateral RLN damage is usually the complication of thyroid cancer surgery; in these instances when the nerve is involved in cancer, unilateral nerve resection or resection and nerve graft have been proposed [27].

Hong et al. [28] performed immediate direct anastomosis of RLNs injured during surgery for thyroid cancer; they found that patients undergoing immediate direct RLN anastomosis demonstrated better phonation and perceptually rated voice quality than those who did not undergo repair.

\subsection{Spinal accessory nerve (SAN)}

The accessory nerve is unique in that its name is based on a historical misunderstanding regarding its origin although it retains its original cranial nerve terminology; but contemporary nomenclature is more inclined toward spinal origin of this nerve, since the cranial part immediately joins the vagus nerve and the spinal part is considered the only and main part of the eleventh nerve [29] (Figure 26).

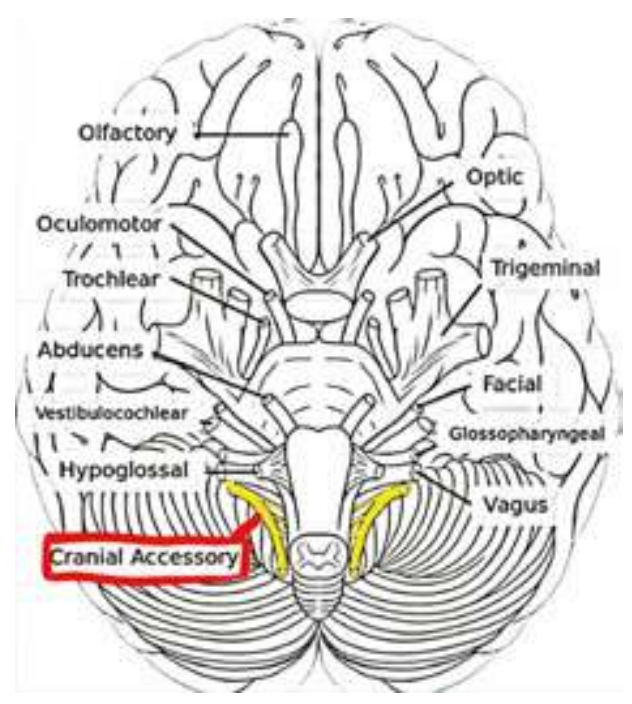

Figure 26. The 11th nerve; as the name implies, some authors think of this nerve as a spinal nerve.

During the past century, the anatomy and blood supply of SAN have been better understood (Figure 27). The importance of almost all of the SAN plexus to head, neck, and upper extremity motor and sensory functions has come to be realized. Because of this understanding, surgical neck dissection has become progressively more conservative toward preserving this nerve as much as possible. 


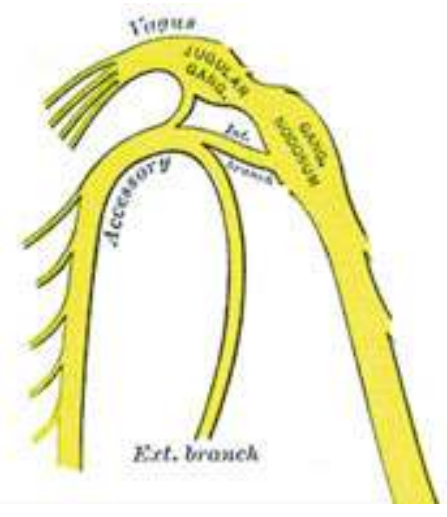

Figure 27. The relations of the SAN to vagus nerve (Gray's anatomy).

Iatrogenic injuries to the spinal accessory are not uncommon during lymph node biopsy of the posterior cervical triangle.

Park et al. review the operative techniques and surgical outcomes of 156 surgical repairs of the SAN following iatrogenic injury during lymph node biopsy procedures. SAN injuries present challenges for surgical exploration and repair because of the nerve's size and location in the Posterior cervical triangle. They concluded that patients with diminished or absent function achieved favorable functional outcomes by corrective surgery. Surgeons performing lymph node biopsy procedures in Zone I of posterior cervical triangle should be aware of the potential risk of injury to the SAN [30].

Nerve transfer between the SAN and the suprascapular nerve is a standard technique in brachial plexus surgery for shoulder reanimation. In cases of global brachial plexus injury, donor nerves are few and at times severely traumatized owing to extensive traction forces. Bhandari and Deb [31] offer the use of the contralateral SAN as an additional option in the reinnervation of an injured Suprascapular nerve in such circumstances.

\subsection{Hypoglossal nerve}

Use of the entire hypoglossal nerve for nerve transfer in obstetric palsy is not recommended because of major donor nerve morbidity in terms of feeding and speech problems (Figure 28).

The hypoglossal nerve to facial nerve transfer is one of the facial reanimation procedures, care must be taken to preserve the hypoglossal nerve for its primary function, and end-to-side nerve transfer is also mentioned in the literature. Beutner et al. have described the modified technique of the hypoglossal-facial-jump anastomosis without an interposition graft [32].

Al-thunyan et al. used a hemi-hypoglossal nerve transfer for biceps reinnervation in obstetric palsy in three infants with multiple root avulsions. 
Elbow flexion was seen in two of the three operated patients with no reported feeding problem. Speech assessment was done at the 20-27 months of age and early speech development was unaffected [33].

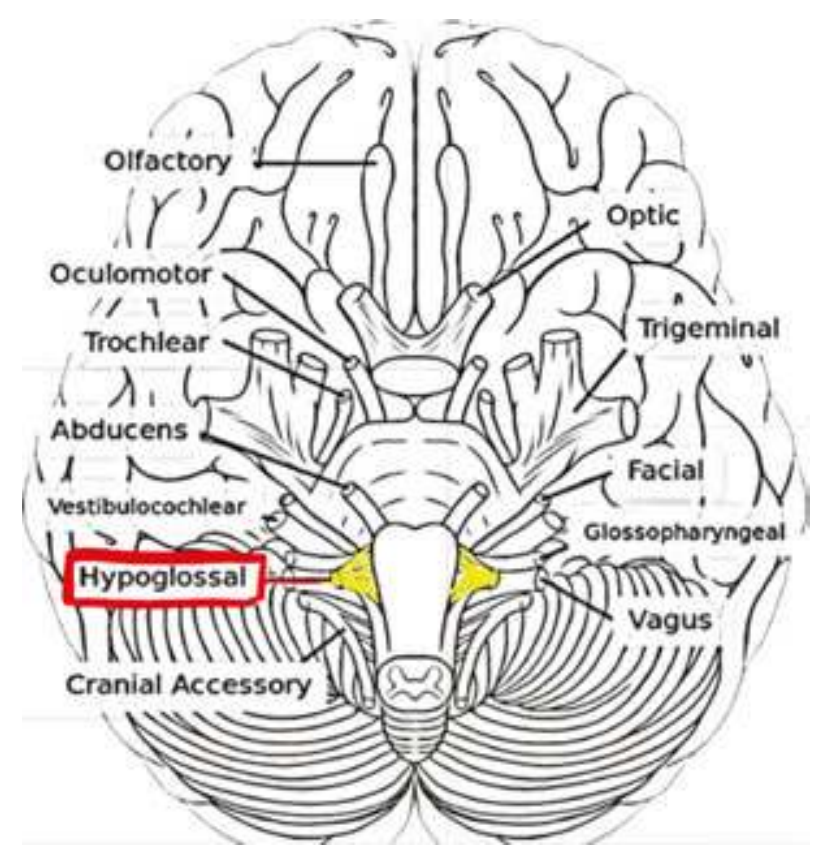

Figure 28. The 12th cranial nerve.

\section{Summary}

The knowledge of cranial nerves' anatomy is an important and integral part of head and neck surgery; we have discussed the cranial nerve anatomy and surgery, ablations, and reconstructions and several personal cranial nerve surgeries have been included in this chapter. The results indicate that patients are at greatest danger of cranial nerve damage during times of stress and that surgeons should take particular care to protect specific nerves in tough conditions.

\section{Acknowledgements}

We wish to thank all the patients who permitted us to show their faces. 


\section{Author details}

Shahram Nazerani ${ }^{1}$ and Tina Nazerani ${ }^{2 *}$

*Address all correspondence to: tnazerani@yahoo.com

1 Rasool Akram General Hospital, Iran Medical University of Medical Sciences, Tehran, Iran

2 General Practitioner, Graz, Austria

\section{References}

[1] Clarke E, Jacyna LS. Nineteenth-century origins of neuroscientific concepts. Berkeley and Los Angeles: University of California Press. 1987.

[2] Shoja et al., 2007; Shaw, 1992.

[3] Flamm ES. Historical observations on the cranial nerves. J Neurosurg 1967;27:285-97 (Flamm, 1967; Shaw, 1992).

[4] Shaw JP. A history of the enumeration of the cranial nerves by European and British anatomists from the time of Galen to 1895, with comments on nomenclature. Clin Anat $1992 ; 5$.

[5] Davis MC, Griessenauer CJ, Bosmia AN, Tubbs RS, Shoja MM. The naming of the cranial nerves: a historical review. Clin Anat 2014;27:14-9

[6] Thiruvenkatarajan V, Van Wijk RM, Rajbhoj A. Cranial nerve injuries with supraglottic airway devices: a systematic review of published case reports and series. Anaesthesia 2015;70:344-59.

[7] Fokkema M, de Borst GJ, Nolan BW, Indes J, Buck DB, Lo RC, Moll FL, Schermerhorn ML, on behalf of the Vascular Study Group of New England. Clinical relevance of cranial nerve injury following carotid endarterectomy. Eur J Vasc Endovasc Surg 2014 Jan;47(1):2e7.

[8] van Riel D, Verdijk R, Kuiken T. The olfactory nerve: a shortcut for influenza and other viral diseases into the central nervous system. J Pathol 2015 Jan;235(2):277-87. doi: 10.1002/path.4461.

[9] Kumar R. Esthesioneuroblastoma: multimodal management and review of literature. World J Clin Cases 2015 Sep; 3(9):774-8. doi: 10.12998/wjcc.v3.i9.774 [published online Sep 16]. 
[10] Coelho DH, Costanzo RM. Posttraumatic olfactory dysfunction. Auris Nasus Larynx 2015 Oct. pii: S0385-8146(15)00200-X. doi: 10.1016/j.anl.2015.08.006 [Epub ahead of print].

[11] Sadagopan KA, Wasserman BN. Managing the patient with oculomotor nerve palsy. Curr Opin Ophthalmol 2013;24(5):438-47. doi: 10.1097/ICU.0b013e3283645a9b.

[12] Bagheri SC, Meyer RA, Khan HA, Kuhmichel A, Steed MB. Retrospective review of microsurgical repair of 222 lingual nerve injuries. J Oral Maxillofac Surg 2010;68(4):71523. doi: 10.1016/j.joms.2009.09.111 [Epub 2009 Dec 29].

[13] Carr MM, Ross DA, Zuker RM. Cranial nerve defects incongenital facial palsy. J Otolaryngol 1997;26:80-7.

[14] Amer TA, El-Minawi HM, El-Shazly MI. Low-level versus high-level placement of gold plates in the upper eyelid in patients with facial palsy. Clin Ophthalmol 2011;5:891-5. doi: 10.2147/OPTH.S21491 [Epub 2011 June 30].

[15] Terzis JK, Karypidis D, Blink restoration in adult facial paralysis. Plast Reconstr Surg 2010;126(1):126-39.

[16] Rose EH. Autogenous fascia lata grafts: clinical applications in reanimation of the totally or partially paralyzed face. Plast Reconstr Surg 2005;116(1):20-32.

[17] Humphrey CD, McIff TE, Sykes KJ, et al. Suture biomechanics and static facial suspension. Arch Facial Plast Surg 2007;9(3):188-93.

[18] Byrne PJ, Kim M, Boahene K, et al. Temporalis tendon transfer as part of a comprehensive approach to facial reanimation. Arch Facial Plast Surg 2007;9(4):234-41.

[19] Terzis JK, Anesti K. Novel use of platysma for oral sphincter substitution or countering excessive pull of a free muscle. J Plast Reconstr Aesthet Surg 2013;66(8):1045-57. doi: 10.1016/j.bjps.2013.04.014 [Epub 2013 May 17].

[20] Terzis JK, Karypidis D. Therapeutic strategies in post-facial paralysis synkinesis in adult patients. Plast Reconstr Surg 2012;129(6):925e-939e. doi: 10.1097/PRS. Ob013e318230e758.

[21] Choi KH, Rho SH, Lee JM, Jeon JH, Park SY, Kim J. Botulinum toxin injection of both sides of the face to treat post-paralytic facial synkinesis. J Plast Reconstr Aesthet Surg 2013;66(8):1058-63.

[22] Mehta RP, Weknick Robinson M, Hadlock TA. Validation of the synkinesis assessment questionnaire. Laryngoscope 2007;117(5):923-6.

[23] Husseman J, Mehta RP. Management of synkinesis. Facial Plast Surg 2008;24(2):242-9.

[24] Vanswearingen J. Facial rehabilitation: a neuromuscular reeducation, patient-centered approach. Facial Plast Surg 2008;24(2):250-9. 
[25] Hwang K, Song JS, Yang SC. Communications between the facial nerve and the vestibulocochlear nerve, the glossopharyngeal nerve, and the cervical plexus. J Craniofac Surg 2015 Oct;26(7):2190-2.

[26] Trinidade A, Philpott CM. Bilateral glossopharyngeal nerve palsy following tonsillectomy: a very rare and difficult complication of a common procedure. J Laryngol Otol 2015;129(4):392-4 [Epub 2015 Feb 20].

[27] Yumoto E, Sanuki T, Kumai Y. Immediate recurrent laryngeal nerve reconstruction and vocal outcome. Laryngoscope 2006;116:1657-61

[28] Hong JW, Roh TS, Yoo HS, Hong HJ, Choi HS, Chang HS, Park CS, Kim YS. Outcome with immediate direct anastomosis of recurrent laryngeal nerves injured during thyroidectomy. Laryngoscope 2014;124:1402-8.

[29] Ryan S, Blyth P, Duggan N, Wild M, Al-Ali S. Is the cranial accessory nerve really a portion of the accessory nerve? Anatomy of the cranial nerves in the jugular foramen. Anatomical Science International/Japanese Association of Anatomists 2007;82(1):1-7.

[30] Park SH, Esquenazi Y, Kline DG, Kim DH. Surgical outcomes of 156 spinal accessory nerve injuries caused by lymph node biopsy procedures. J Neurosurg Spine 2015;23(4): 518-25. doi: 10.3171/2014.12.SPINE14968. [Epub 2015 June 26]

[31] Bhandari PS, Deb P. Use of contralateral spinal accessory nerve for ipsilateral suprascapular neurotization in global brachial plexus injury: a new technique. J Neurosurg Spine 2016;24(1):186-8. doi: 10.3171/2015.4.SPINE15108 [Epub 2015 September 25].

[32] Beutner D, Luers JC, Grosheva M. Hypoglossal-facial-jump-anastomosis without an interposition nerve graft. Laryngoscope 2013;123(10):2392-6. doi: 10.1002/lary.24115 [Epub 2013 May 13].

[33] Al-Thunyan A, Al-Qattan MM, Al-Meshal O, Al-Husainan H, Al-Assaf A. Hemihypoglossal nerve transfer for obstetric brachial plexus palsy: report of 3 cases. J Hand Surg Am 2015;40(3):448-51. doi: 10.1016/j.jhsa.2014.11.018 [Epub 2015 January 21]. 
